\title{
Some Properties for Strong Differential Subordination of Analytic Functions Involving Ruscheweyh Derivative Operator
}

\section{Asraa Abdul Jaleel Husien}

Technical Institute, Diwaniya, Al-Furat Al-Awsat Technical University, Iraq e-mail: asraalsade2@gmail.com

\section{Abstract}

In this paper, we introduce and study some properties for strong differential subordinations of analytic functions associated with Ruscheweyh derivative operator defined in the open unit disk and closed unit disk of the complex plane.

\section{Introduction}

Let $U=\{z \in \mathbb{C}:|z|<1\}$ and $\bar{U}=\{z \in \mathbb{C}:|z| \leq 1\}$ denote the open unit disk and the closed unit disk of the complex plane, respectively. Let $\mathcal{H}(U \times \bar{U})$ the class of all analytic functions in $U \times \bar{U}$. For $n \in \mathbb{N}=\{1,2, \ldots\}$ and $a \in \mathbb{C}$, let $\mathcal{H}^{*}[a, n, \zeta]=\{f \in$ $\left.\mathcal{H}(U \times \bar{U}): f(z, \zeta)=a+a_{n}(\zeta) z^{n}+a_{n+1}(\zeta) z^{n+1}+\cdots, z \in U, \zeta \in \bar{U}\right\}$, where $a_{k}(\zeta)$ are holomorphic functions in $\bar{U}$ for $k \geq n$.

Also, let $\mathcal{A}_{n \zeta}^{*}=\left\{f \in \mathcal{H}(U \times \bar{U}): f(z, \zeta)=z+a_{n+1}(\zeta) z^{n+1}+\cdots, z \in U, \zeta \in \bar{U}\right\}$, where $a_{k}(\zeta)$ are holomorphic functions in $\bar{U}$ for $k \geq n+1$.

A function $f \in \mathcal{H}^{*}[a, n, \zeta]$ is said to be starlike in $U \times \bar{U}$ if

Received: February 14, 2020; Accepted: April 2, 2020

2010 Mathematics Subject Classification: 30C45.

Keywords and phrases: analytic functions, convex functions, strong differential subordinations, Ruscheweyh derivative operator.

Copyright () 2020 Asraa Abdul Jaleel Husien. This is an open access article distributed under the Creative Commons Attribution License, which permits unrestricted use, distribution, and reproduction in any medium, provided the original work is properly cited. 


$$
\operatorname{Re}\left\{\frac{z f_{z}^{\prime}(z, \zeta)}{f(z, \zeta)}\right\}>0, \quad(z \in U, \zeta \in \bar{U})
$$

and convex in $U \times \bar{U}$ if

$$
\operatorname{Re}\left\{\frac{z f^{\prime \prime}(z, \zeta)}{f_{z}^{\prime}(z, \zeta)}+1\right\}>0, \quad(z \in U, \zeta \in \bar{U})
$$

Denote the classes of starlike and convex functions in $U \times \bar{U}$ by $S_{\zeta}^{*}$ and $K_{\zeta}^{*}$, respectively.

Let $\mathcal{A}_{\zeta}^{*}$ denote the subclass of the functions $f(z, \zeta) \in \mathcal{H}(U \times \bar{U})$ of the form:

$$
f(z, \zeta)=z+\sum_{k=2}^{\infty} a_{k}(\zeta) z^{k}, \quad z \in U, \zeta \in \bar{U}
$$

which are analytic and univalent in $U \times \bar{U}$.

The Ruscheweyh derivative operator $\mathcal{R}^{\lambda}: \mathcal{A}_{\zeta}^{*} \rightarrow \mathcal{A}_{\zeta}^{*}$ (see [7]) is defined by

$$
\mathcal{R}^{\lambda} f(z, \zeta)=z+\sum_{k=2}^{\infty} \frac{\Gamma(\lambda+k)}{\Gamma(\lambda+1) \Gamma(k)} a_{k}(\zeta) z^{k} \quad\left(\lambda \in \mathbb{N}_{0}=\mathbb{N} \bigcup\{0\}\right)
$$

It is easy to obtain from (1.2) that

$$
z\left(\mathcal{R}^{\lambda} f(z, \zeta)\right)_{z}^{\prime}=(\lambda+1) \mathcal{R}^{\lambda+1} f(z, \zeta)-\lambda \mathcal{R}^{\lambda} f(z, \zeta)
$$

In recent years, many authors obtained various interesting results associated with strong differential subordination and superordination for example (see [1, 2, 3, 8, 9, 10, 11]).

In order to derive our main results, we need the following definition and lemmas.

Definition 1.1 [6]. Let $f(z, \zeta), g(z, \zeta)$ be analytic in $U \times \bar{U}$. The function $f(z, \zeta)$ is said to be strongly subordinate to $g(z, \zeta)$, written $f(z, \zeta) \prec \prec F(z, \zeta), z \in U$, $\zeta \in \bar{U}$, if there exists an analytic function $w$ in $U$ with $w(0)=0$ and $|w(z)|<1, z \in U$ such that $f(z, \zeta)=g(w(z), \zeta)$ for all $\zeta \in \bar{U}$. 
Lemma 1.1 [5]. Let $h(z, \zeta)$ be a convex function with $h(0, \zeta)=a$, for every $\zeta \in \bar{U}$ and let $\gamma \in \mathbb{C}^{*}=\mathbb{C} \backslash\{0\}$ with $\operatorname{Re}(\gamma) \geq 0$. If $p \in \mathcal{H}^{*}[a, n, \zeta]$ and

$$
p(z, \zeta)+\frac{1}{\gamma} z p_{z}^{\prime}(z, \zeta) \prec \prec h(z, \zeta), \quad(z \in U, \zeta \in \bar{U}),
$$

then

$$
p(z, \zeta) \prec \prec q(z, \zeta) \prec \prec h(z, \zeta), \quad(z \in U, \zeta \in \bar{U}),
$$

where $q(z, \zeta)=\frac{\gamma}{\underline{\gamma}} \int_{0}^{z} t^{\frac{\gamma}{n}-1} h(t, \zeta) d t$ is convex and it is the best dominant of (1.4). $n z^{n}$

Lemma 1.2 [4]. Let $q(z, \zeta)$ be a convex function in $U \times \bar{U}$ for all $\zeta \in \bar{U}$ and let $h(z, \zeta)=q(z, \zeta)+n \delta z q_{z}^{\prime}(z, \zeta), \quad z \in U, \quad \zeta \in \bar{U}$, where $\delta>0$ and $n$ is a positive integer. If

$$
p(z, \zeta)=q(0, \zeta)+p_{n}(\zeta) z^{n}+p_{n+1}(\zeta) z^{n+1}+\cdots,
$$

is analytic in $U \times \bar{U}$ and

$$
p(z, \zeta)+\delta z p_{z}^{\prime}(z, \zeta) \prec \prec h(z, \zeta), \quad(z \in U, \zeta \in \bar{U}),
$$

then

$$
p(z, \zeta) \prec \prec q(z, \zeta), \quad(z \in U, \zeta \in \bar{U}),
$$

and this result is sharp.

\section{Main Results}

Theorem 2.1. Let $h(z, \zeta)$ be a convex function such that $h(0, \zeta)=1$. If $f \in A_{\zeta}^{*}$ satisfies the strong differential subordination:

$$
\left(\mathcal{R}^{\lambda} f(z, \zeta)\right)_{z}^{\prime} \prec \prec h(z, \zeta)
$$

then

$$
\frac{\mathcal{R}^{\lambda} f(z, \zeta)}{z} \prec \prec q(z, \zeta) \prec \prec h(z, \zeta),
$$


where $q(z, \zeta)=\frac{1}{z} \int_{0}^{z} h(t, \zeta) d t$ is convex and it is the best dominant.

Proof. Suppose that

$$
p(z, \zeta)=\frac{\mathcal{R}^{\lambda} f(z, \zeta)}{z}, \quad z \in U, \zeta \in \bar{U}
$$

Then the function $p(z, \zeta)$ is analytic in $U \times \bar{U}$ and $p(0, \zeta)=1$.

Simple computations from (2.2), we get

$$
p(z, \zeta)+z p_{z}^{\prime}(z, \zeta)=\left(\mathcal{R}^{\lambda} f(z, \zeta)\right)_{z}^{\prime} .
$$

Using (2.3), (2.1) becomes

$$
p(z, \zeta)+z p_{z}^{\prime}(z, \zeta) \prec \prec h(z, \zeta)
$$

An application of Lemma 1.1 with $n=1, \gamma=1$ yields

$$
\frac{\mathcal{R}^{\lambda} f(z, \zeta)}{z} \prec \prec q(z, \zeta)=\frac{1}{z} \int_{0}^{z} h(t, \zeta) d t \prec \prec h(z, \zeta) .
$$

By taking $h(z, \zeta)=\frac{\zeta+(2 \rho-\zeta) z}{1+z}, 0 \leq \rho<1$ in Theorem 2.1, we obtain the following corollary:

Corollary 2.1. If $f \in A_{\zeta}^{*}$ satisfies the strong differential subordination:

$$
\left(\mathcal{R}^{\lambda} f(z, \zeta)\right)_{z}^{\prime} \prec \prec \frac{\zeta+(2 \rho-\zeta) z}{1+z},
$$

then

$$
\frac{\mathcal{R}^{\lambda} f(z, \zeta)}{z} \prec \prec \frac{1}{z} \int_{0}^{z} \frac{\zeta+(2 \rho-\zeta) t}{1+t} d t=2 \rho-\zeta+\frac{2(\zeta-\rho)}{z} \ln (1+z)
$$

Theorem 2.2. Let $q(z, \zeta)$ be a convex function such that $q(0, \zeta)=1$ and let $h$ be the function $h(z, \zeta)=q(z, \zeta)+z q_{z}^{\prime}(z, \zeta)$. If $f \in \mathcal{A}_{\zeta}^{*}$ satisfies the strong differential subordination: 


$$
\left(\frac{z \mathcal{R}^{\lambda+1} f(z, \zeta)}{\mathcal{R}^{\lambda} f(z, \zeta)}\right)_{z}^{\prime} \prec \prec h(z, \zeta),
$$

then

$$
\frac{\mathcal{R}^{\lambda+1} f(z, \zeta)}{\mathcal{R}^{\lambda} f(z, \zeta)} \prec \prec q(z, \zeta) .
$$

Proof. Suppose that

$$
p(z, \zeta)=\frac{\mathcal{R}^{\lambda+1} f(z, \zeta)}{\mathcal{R}^{\lambda} f(z, \zeta)}, \quad z \in U, \zeta \in \bar{U} .
$$

Then the function $p(z, \zeta)$ is analytic in $U \times \bar{U}$ and $p(0, \zeta)=1$.

Differentiating both sides of (2.5) with respect to $z$ and using (2.4), we have

$$
\begin{aligned}
& p(z, \zeta)+z p_{z}^{\prime}(z, \zeta) \\
= & \frac{\mathcal{R}^{\lambda+1} f(z, \zeta)}{\mathcal{R}^{\lambda} f(z, \zeta)}+\frac{\mathcal{R}^{\lambda} f(z, \zeta)\left(\mathcal{R}^{\lambda+1} f(z, \zeta)\right)_{z}^{\prime}-\mathcal{R}^{\lambda+1} f(z, \zeta)\left(\mathcal{R}^{\lambda} f(z, \zeta)\right)_{z}^{\prime}}{\left[\mathcal{R}^{\lambda} f(z, \zeta)\right]^{2}} \\
= & \frac{\mathcal{R}^{\lambda} f(z, \zeta)\left(z \mathcal{R}^{\lambda+1} f(z, \zeta)\right)_{z}^{\prime}-z \mathcal{R}^{\lambda+1} f(z, \zeta)\left(\mathcal{R}^{\lambda} f(z, \zeta)\right)_{z}^{\prime}}{\left[\mathcal{R}^{\lambda} f(z, \zeta)\right]^{2}} \\
= & \left(\frac{z \mathcal{R}^{\lambda+1} f(z, \zeta)}{\mathcal{R}^{\lambda} f(z, \zeta)}\right)_{z}^{\prime} \prec \prec h(z, \zeta) .
\end{aligned}
$$

An application of Lemma 1.2, we obtain

$$
\frac{\mathcal{R}^{\lambda+1} f(z, \zeta)}{\mathcal{R}^{\lambda} f(z, \zeta)} \prec \prec q(z, \zeta) .
$$

Theorem 2.3. Let $q(z, \zeta)$ be a convex function such that $q(0, \zeta)=1$ and let $h$ be the function $h(z, \zeta)=q(z, \zeta)+\frac{1}{\lambda+2} z q_{z}^{\prime}(z, \zeta)$, where $\lambda+1>0$. Suppose that

$$
F(z, \zeta)=\frac{\lambda+2}{z^{\lambda+1}} \int_{0}^{z} t^{\lambda} f(t, \zeta) d t, \quad z \in U, \zeta \in \bar{U}
$$


If $f \in \mathcal{A}_{\zeta}^{*}(p)$ satisfies the strong differential subordination

$$
\left(\mathcal{R}^{\lambda} f(z, \zeta)\right)_{z}^{\prime} \prec \prec h(z, \zeta)
$$

then

$$
\left(\mathcal{R}^{\lambda} F(z, \zeta)\right)_{z}^{\prime} \prec \prec q(z, \zeta)
$$

Proof. Suppose that

$$
p(z, \zeta)=\left(\mathcal{R}^{\lambda} F(z, \zeta)\right)_{z}^{\prime}, \quad z \in U, \zeta \in \bar{U}
$$

Then the function $p(z, \zeta)$ is analytic in $U \times \bar{U}$ and $p(0, \zeta)=1$.

From (2.7), we have

$$
z^{\lambda+1} F(z, \zeta)=(\lambda+2) \int_{0}^{z} t^{\lambda} f(t, \zeta) d t
$$

Differentiating both sides of (2.10) with respect to $z$, we get

$$
(\lambda+2) f(z, \zeta)=(\lambda+1) F(z, \zeta)+z F_{z}^{\prime}(z, \zeta)
$$

and

$$
(\lambda+2) \mathcal{R}^{\lambda} f(z, \zeta)=(\lambda+1) \mathcal{R}^{\lambda} F(z, \zeta)+z\left(\mathcal{R}^{\lambda} F(z, \zeta)\right)_{z}^{\prime}
$$

So

$$
\left(\mathcal{R}^{\lambda} f(z, \zeta)\right)_{z}^{\prime}=\left(\mathcal{R}^{\lambda} F(z, \zeta)\right)_{z}^{\prime}+\frac{z\left(\mathcal{R}^{\lambda} F(z, \zeta)\right)_{z}^{\prime \prime}}{\lambda+2}
$$

From (2.9) and (2.11), we obtain

$$
p(z, \zeta)+\frac{1}{\lambda+2} z p_{z}^{\prime}(z, \zeta)=\left(\mathcal{R}^{\lambda} f(z, \zeta)\right)_{z}^{\prime} .
$$

Using (2.12), (2.8) becomes

$$
p(z, \zeta)+\frac{1}{\lambda+2} z p_{z}^{\prime}(z, \zeta) \prec \prec q(z, \zeta)+\frac{1}{\lambda+2} z p_{z}^{\prime}(z, \zeta) .
$$

An application of Lemma 1.2 yields $p(z, \zeta) \prec \prec q(z, \zeta)$. By using (2.8), we obtain 


$$
\left(\mathcal{R}^{\lambda} F(z, \zeta)\right)_{z}^{\prime} \prec \prec q(z, \zeta)
$$

Theorem 2.4. Let $h(z, \zeta)$ be a convex function such that $h(0, \zeta)=1$. If $0 \leq \sigma<p$, $\theta \in \mathbb{C}$ and $f \in A_{\zeta}^{*}$ satisfies the strong differential subordination:

$$
\frac{1-\theta}{1-\sigma}\left(\frac{\mathcal{R}^{\lambda} f(z, \zeta)}{z}-\sigma\right)+\frac{\theta}{1-\sigma}\left(\left(\mathcal{R}^{\lambda} f(z, \zeta)\right)_{z}^{\prime}-\sigma\right) \prec \prec h(z, \zeta),
$$

then

$$
\frac{1}{1-\sigma}\left(\frac{R^{\lambda} f(z, \zeta)}{z}-\sigma\right) \prec \prec q(z, \zeta) \prec \prec h(z, \zeta),
$$

where $q(z, \zeta)=\frac{1}{\theta} z^{-\frac{1}{\theta}} \int_{0}^{z} t^{\frac{1}{\theta}-1} h(t, \zeta) d t$ is convex and it is the best dominant.

Proof. Suppose that

$$
p(z, \zeta)=\frac{1}{1-\sigma}\left(\frac{\mathcal{R}^{\lambda} f(z, \zeta)}{z}-\sigma\right), \quad z \in U, \zeta \in \bar{U}
$$

Then the function $p(z, \zeta)$ is analytic in $U \times \bar{U}$ and $p(0, \zeta)=1$.

Differentiating both sides of (2.14) with respect to $z$, we have

$$
p(z, \zeta)+\theta z p_{z}^{\prime}(z, \zeta)=\frac{1-\theta}{1-\sigma}\left(\frac{\mathcal{R}^{\lambda} f(z, \zeta)}{z}-\sigma\right)+\frac{\theta}{1-\sigma}\left(\left(\mathcal{R}^{\lambda} f(z, \zeta)\right)_{z}^{\prime}-\sigma\right)
$$

From (2.13) and (2.15), we get

$$
p(z, \zeta)+\theta z p_{z}^{\prime}(z, \zeta) \prec \prec h(z, \zeta) .
$$

An application of Lemma 1.1 with $n=1, \gamma=\frac{1}{\theta}$ yields

$$
\frac{1}{1-\sigma}\left(\frac{\mathcal{R}^{\lambda} f(z, \zeta)}{z}-\sigma\right) \prec \prec q(z, \zeta)=\frac{1}{\theta} z^{-\frac{1}{\theta}} \int_{0}^{z} t^{\frac{1}{\theta}-1} h(t, \zeta) d t \prec \prec h(z, \zeta)
$$




\section{References}

[1] N. E. Cho, O. S. Kwon and H. M. Srivastava, Strong differential subordination and superordination for multivalently meromorphic functions involving the Liu-Srivastava operator, Integral transforms Spec. Funct. 21(8) (2010), 589-601. https://doi.org/10.1080/10652460903494751

[2] M. P. Jeyaraman and T. K. Suresh, Strong differential subordination and superordination of analytic functions, J. Math. Anal. Appl. 385(2) (2012), 854-864. https://doi.org/10.1016/j.jmaa.2011.07.016

[3] A. A. Lupas, A note on strong differential superordinations using a generalized Sălăgean operator and Ruscheweyh operator, Stud. Univ. Babes-Bolyai Math. 57(2) (2012), 135165.

[4] S. S. Miller and P. T. Mocanu, On some classes of first-order differential subordinations, Michigan Math. J. 32(2) (1985), 185-195. https://doi.org/10.1307/mmj/1029003185

[5] S. S. Miller and P. T. Mocanu, Differential Subordinations: Theory and Applications, Series on Monographs and Textbooks in Pure and Applied Mathematics, Vol. 225, Marcel Dekker Inc., New York and Basel, 2000. https://doi.org/10.1201/9781482289817

[6] G. I. Oros and Gh. Oros, Strong differential subordination, Turk. J. Math. 33 (2009), 249257.

[7] S. Ruscheweyh, New criteria for univalent functions, Proc. Amer. Math. Soc. 49 (1975), 109-115. https://doi.org/10.1090/S0002-9939-1975-0367176-1

[8] A. K. Wanas and B. A. Frasin, Strong differential sandwich results for Frasin operator, Earthline J. Math. Sci. 3(1) (2020), 95-104. https://doi.org/10.34198/ejms.3120.95104

[9] A. K. Wanas and A. Alb Lupas, On a new strong differential subordinations and superordinations of analytic functions involving the generalized differential operator, Int. J. Pure Appl. Math. 116(3) (2017), 571-579.

[10] A. K. Wanas and A. H. Majeed, Strong differential subordinations for higher-order derivatives of multivalent analytic functions defined by linear operator, Khayyam J. Math. 3(2) (2017), 160-171.

[11] A. K. Wanas and A. H. Majeed, Strong differential subordination and superordination of meromorphic multivalent quasi-convex functions, Kragujevac J. Math. 44(1) (2020), 27-39. 